\title{
Personalised medical management of patients with melanoma (part 1)
}

\author{
Justyna Gil', Izabela Łaczmańska1,2, Maria M. Sąsiadek', Marcin Ziętek³,4
}

\author{
'Chair and Department of Genetics, Faculty of Medicine, Wroclaw Medical University, Wroclaw, Poland \\ 2Department of Molecular Diagnostics of Cancer, Wroclaw Comprehensive Cancer Centre, Wroclaw, Poland \\ ${ }^{3}$ Department of Surgical Oncology, Department of Oncology, Wroclaw Medical University, Wroclaw, Poland \\ ${ }^{4}$ Surgical Oncology Ward, Wroclaw Comprehensive Cancer Centre, Wroclaw, Poland
}

In recent years, a dynamic increase has been observed in occurrence of melanomas, especially in young and middle-aged patients. This is the reason why curing these patients has become a priority also in the economic context. Melanomas belong to a group of neoplasms of very high genetic heterogeneity. The most common genetic alterations concern two signalling pathways: mitogen-activated pathway (MAPK) and phosphoinositide 3-kinase (PI3K) pathway. Identification of the characteristic molecular changes in the neoplastic tissue allows optimisation and individualisation of the therapy. Thus, it contributes to an increase in successful cancer treatment, reduction of treatment side effects and to improvement of the patients' quality of life. Currently, the standard management of skin melanoma patients involves - along with surgical treatment and classical chemo/radiotherapy which is now less frequently used - also introduction of targeted therapy focused on molecular changes within the tumour tissue as well as immunotherapy which relies on activating the immune system.

Key words: melanoma, BRAF, NRAS, targeted therapy

\section{Introduction}

Individualised oncological therapy involves therapeutic management aimed at selecting the treatment to obtain maximum benefits while minimising side effects. Effectiveness of such treatment is always associated with evaluation of the patient by a multidisciplinary team of clinicians. The objective of this assessment is to determine optimal therapeutic approach ("tailored treatment"). Implementation of such procedure is possible due to immense technological development observed in genetics and molecular biology over the last decade. It involves:

- introduction of a new classification of neoplasms,

- search for new therapeutic goals,
- assessment of the patient's response to treatment (pharmacogenomics),

- detecting treatment resistance,

- detecting recurrence at a very early stage,

- cancer risk assessment [1].

\section{Epidemiology and risk factors}

Melanoma is one of those cancers in which targeted treatment has been used for several years. It is a malignant neoplasm originating from melanocytes, i.e. cells that produce the pigment called melanin. These are cells of neuroectodermal origin [2]. The most common primary location of melanoma is the skin (over $96 \%$ of cases), especially surfaces exposed to sunlight. Other

\section{How to cite:}

Gil J, Łaczmańska I, Sąsiadek MM, Ziętek M. Personalised medical management of patients with melanoma (part 1). NOWOTWORY J Oncol 2021; 71: 169-175. 
locations that are much less common include: the conjunctiva and uvea, oral, pharyngeal and genital mucosa, meninges and distal parts of the body (including subungual localization) [2].

The highest incidence of melanoma is observed in Australia, New Zealand and North America. In Poland, melanoma is relatively rare, and its standardized incidence rate is approximately 6.5 per 100,000. According to the National Cancer Registry of 2017, there were 3,785 cases of melanoma in Poland recorded (1,796 men and 1,989 women), and 1,410 deaths caused by melanoma [3]. Although melanoma is a rare neoplasm (about 2\% of all cancers), the last dozen years witnessed a dynamic increase of its incidence, especially in the Caucasian population. This is also the case in Poland (according to the data of the National Cancer Registry, an increase of over $70 \%$ in 10 years). The relatively young age of onset (30-50 years) is also important, as it translates significantly to serious socio-economic consequences [4]. Importantly, melanoma-related mortality in Poland is 20\% higher that the respective number in western countries, even though the morbidity rate in Poland is lower. This is clearly reflected in statistical data concerning differences in treatment effectiveness in individual countries - among all cancers, the differences for melanoma are the highest (Poland 69.8 vs. Germany 93.1) [3]. It is probably related to late detection / diagnosis of the disease and ignorance and/or failure to apply prophylaxis.

The rapid growth rate and high metastatic potential place melanoma among those cancers which are the most difficult to treat and have the worst prognosis [2]. Therefore, it is very important to diagnose the disease quickly and accurately, because if the cancer is detected early (when it is locally limited to its primary focus), it is almost $100 \%$ curable - it can be removed surgically [2].

The main risk factor for development of melanoma is light skin and exposure to ultraviolet (UV) radiation, either natural (especially UV-B), or artificial (indoor tanning, especially UV-A radiation). People who have been exposed to UV radiation intensively and intermittently, and who have suffered sunburns during childhood and/or adolescence, are at an increased risk of developing this disease, compared to those who have experienced long-term and regular UV exposure. People who have suffered more than 5 severe episodes of sunburn are approximately 2 times more likely to develop melanoma [5].

There is a gradient in the incidence of melanoma in Europe - the highest rate of morbidity is recorded in the north of the continent, while in the south there are significantly fewer cases. Fewer melanoma patients in southern European populations are likely associated with chronic sun exposure (compared to periodic / sporadic exposure in the north) and characteristically darker skin phototype which provides natural protection against UV radiation [6].

\section{Diagnostics}

Most melanomas form de novo - about $50-60 \%$ on the skin without pigmentation changes, and about $40 \%$ arise on exi- sting pigmentary lesions [4]. Self-observation of skin changes, especially atypical pigmented nevi, is extremely important, and any disturbing change should be reported to a specialist. Patients may use the ABCDE scale for nevus assessment and initial identification of some melanomas, with letters corresponding to lesion features:

- A - asymmetry of the nevus,

- B-border irregularity,

- C - colour inconsistencies,

- D - diameter larger than $5 \mathrm{~mm}$,

- E - evolution / elevation, marking a change in shape or protuberance of the nevus over time [7].

Lesions may be associated with ulceration and/or bleeding. A dermatoscopy by a specialist is the basis for a clinical diagnosis of the disease. Subsequently, the suspicious lesion should be surgically removed with a minimum margin of 1-2 mm of healthy skin and subjected to histopathological analysis for diagnosis. There are 4 main histological subtypes of melanoma:

- superficial spreading melanoma - SSM (41\%),

- nodular melanoma - NM (16\%),

- melanoma arising from the lentigo (lentigo maligna melanoma - LMM) (2.7-14\%), and

- subungual or limb melanoma (acral lentiginous melanoma - ALM) (7-10\%).

Other rarer types include desmoplastic melanoma or blue nevus melanoma $[7,8]$. The histopathology report should include subtype diagnosis, as well as other important features:

- macroscopic characteristics, i.e. size of the excised skin fragment with the lesion, location of the lesion on the skin, tumour dimensions and lesion description (including its colour, border, nodule or its absence, satellite foci),

- microscopic features, i.e. tumour thickness in mm (Breslow measurement), ulceration or its absence, number of mitoses per $\mathrm{mm}^{2}$, presence or absence of microsatellites and additionally growth phases (radial vs. vertical), presence or absence of lymphocytic infiltration, presence or absence of infiltration of lymphatic vessels, presence or absence of infiltration of nerve trunks $[7,8]$.

Subsequent diagnostic tests (chest X-ray, abdominal ultrasound, lymph node ultrasound, CT or PET) allow staging of the disease advancement. Such comprehensive diagnostics enables forecasting further natural course of the disease (prognostic factor). On the other hand, molecular tests for the presence of mutations within tumours at high clinical stages allow to plan the most effective treatment (predictive factor). There are five stages of clinical tumour advancement [7]:

- Grade 0 - referred to as melanoma in situ-a form that does not exceed the epidermis with no infiltration.

- Grade I - if the melanoma is ulcerated and its thickness does not exceed $1 \mathrm{~mm}$, or if it is not ulcerated and its thickness does not exceed $2 \mathrm{~mm}$, lymph nodes are not affected (NO) and there are no distant metastases (MO). 
- Grade II - it has three subgrades, distinguished by the primary lesion's thickness:

- IIA - if the melanoma is ulcerated and its thickness does not exceed $2 \mathrm{~mm}$ or if it is not ulcerated and its thickness does not exceed $4 \mathrm{~mm}$,

- $\quad$ IIA - if the melanoma is ulcerated and its thickness does not exceed $4 \mathrm{~mm}$ or if it is not ulcerated and its thickness exceeds $4 \mathrm{~mm}$,

- $\quad$ IIC - if the melanoma is ulcerated and its thickness exceeds $4 \mathrm{~mm}$.

In grade II, lymph nodes are not affected (NO) and there are no distant metastases (M0).

- Grade III - presence of metastases in the regional lymph nodes. There are four subgrades (IIIA-IIID) depending on the number of lymph nodes involved and the type of metastasis (micrometastases diagnosed microscopically vs. macrometastases found in a clinical examination). No distant metastases (M0). At this grade, skin metastases are possible in the form of satellite or in-transit foci which can be isolated or associated with metastases to regional lymph nodes.

- Grade IV - the most advanced stage of the disease, characterised by metastases to:

- extra-regional lymph nodes, skin or subcutaneous tissue,

- visceral organs such as the lungs and liver,

- central nervous system - this group of patients has the worst prognosis.

\section{Hereditary / genetic predisposition to melanoma}

In addition to the most common sporadic form of melanoma, hereditary forms are also known. No single inheritance mode has been identified with respect to genetic factors determining melanoma development predisposition, and the familial cases of melanoma have multi-gene background, frequently associated with a specific complexion (light skin with freckles and red hair is associated with higher risk), as well as family habits (e.g. overexposition to solar radiation) [9]. The CDKN2A gene (cyclin-dependent kinase inhibitor 2A), well-studied in the Polish population, is one of the leading and most researched predisposing genes. It encodes the 16 cell cycle control protein (INK4A) and the p14 (ARF) isoform [10]. This gene is located on the short arm of chromosome 9 (9p21). The most common constitutional variant, i.e. the variant which is present in all cells of the body, is c.442G > A (p.A148T, missense type change, substitution of alanine by threonine), which increases the risk of melanoma 2-2.5 times, it also increases the risk of pancreatic, lung, colorectal and breast cancers and malignant tumours of the brain [10-12]. Although the alteration itself does not cause dysfunction of the protein encoded by this gene, it has been suggested that it may be inherited together with another variant that has negative impact on the protein and thus modulates the risk of developing the disease [10].
Interestingly, the data in the ClinVar database do not support the pathogenicity of this lesion and classify it as a benign variant which is not related to a disease. Therefore, the diagnostic result obtained for a given patient should be interpreted in relation to clinical data (including data on the ethnicity of the patient), literature data and emerging new guidelines.

\section{Selected genetic syndromes with an increased risk of melanoma}

There are various genetic syndromes which are associated with the increased risk of development of skin cancers, including melanoma. The greatest risk is observed in xeroderma pigmentosum (XP) and dysplastic nevus syndrome. Li-Fraumeni syndrome predisposes to the development of this cancer to a smaller extent.

Xeroderma pigmentosum is a very rare heterogenic disease characterised by autosomal recessive inheritance. In this disease, the skin displays characteristically increased susceptibility to ultraviolet radiation, which involves high risk of early-age development of skin cancers. The genetic background of $\mathrm{XP}$ involves mutations in the genes which encode enzymes from nucleotide excision repair (NER) responsible for repairing DNA damage caused by UV radiation. The exception is the XPV subtype, in which the disease is caused by mutations in the polymerase $\eta$. There are several sub-types of XP depending on the gene affected by the mutation $(X P A, X P B, X C P, X P D, X P E$, $X P G, E R C(4, D D B 2$ and $P O L H)$. XP prophylaxis involves avoiding exposure to UV radiation, frequent dermatological check-ups and removal of precancerous lesions [13].

Dysplastic nevus syndrome (familial atypical multi mole melanoma syndrome - FAMMM) is inherited in an autosomal dominant manner with variable expression and incomplete penetrance [14]. Apart from melanoma, increased risk of other malignancies is observed, including pancreatic cancer. The risk of developing melanoma in patients with dysplastic nevi is primarily related to the total number of nevi and family history of melanoma [15]. The syndrome is caused by the mutations in genes encoding proteins that regulate the cell cycle, including CDKN2A and CDK4 (cyclin-dependent kinase 4) [15].

Li-Fraumeni syndrome which is the hereditary predisposition to a broad spectrum of neoplasms, is based on a mutation in the suppressor gene (anti-oncogene) TP53 (tumour protein p53). This is an autosomally dominantly inherited syndrome. About $50 \%$ of mutation carriers develop tumours by the age of 30, often multifocal or bilateral lesions. The most common neoplasms associated with Li-Fraumeni syndrome are sarcomas and osteosarcomas, as well as breast cancer, adrenal cortex cancer and malignant tumours of the brain. Melanoma does not belong to the main spectrum of neoplasms found in this syndrome, but the risk of its development is increased. Therefore, prophylaxis should include the analysis of any new skin lesion / nevus by a dermatologist and limitation of exposure to UV radiation [16]. 


\section{Genetic counselling in patients with hereditary melanoma predisposition}

Patients with oncological diseases should be consulted by a clinical geneticist, who should assess whether the disease meets the hereditary cancer syndrome criteria. There are features allowing for such diagnosis even without finding the germline mutation, e.g. diagnosis of the hereditary breast cancer syndrome (HBC-syndrome).

The case is similar for patients with clinical history of melanoma. In families with hereditary predisposition, cancers are diagnosed in young patients (below 40 years of age) and in several close relatives. In the case of melanoma, it should be remembered that development of this cancer may be also associated with shared environmental risk.

As it has been mentioned before, the genetic background in families with accumulation of melanomas is not easily found. Apart from the already mentioned CDKN2A and CDK4 genes, involvement of other genes, of moderate penetrance, has been suggested, too: TERT (telomerase reverse transcriptase), MITF (microphthalmia-associated transcription factor gene), POT1 (protection of telomeres 1) or BAP1 (BRCA1 associated protein 1) [17]. Additionally, genetic counselling should take into account the increased risk of pancreatic cancer (in carriers of CDKN2A mutations). The patient's skin phenotype and geographic origin are important, too, as the risk can differ between particular populations, even in carriers of the same genetic variant. Genetic tests which can be applied to analyse the genetic burden may concern only the c.442G > A (p.A148T) variant in the CDKN2A gene (especially for the Polish population). The gold standard in this type of testing involves sequencing with Sanger method, especially that this method is not very expensive, but has very high sensitivity. The gene fragment that contains the change is amplified by PCR (polymerase chain reaction) and then every nucleotide in the sequence is read by the sequencing reaction. In families with hereditary predisposition, if the p.A148T variant is not detected, sequencing should cover the entire CDKN2A gene including promoter sequence. If no variants are found in the CDKN2A gene, then a test is typically performed for mutations in the CDK4 gene and other genes of potential relevance in melanoma. However, these are informal recommendations, as so far, no guidelines for the analysis of genes other than CDKN2A have been published [18, 19]. Also in other cancer syndromes, such as Lynch syndrome type II, Cowden syndrome, familial retinoblastoma, one should remember about the increased risk of melanoma [18].

\section{Most common molecular changes in melanomas}

Melanomas are a very heterogeneous group of cancers in terms of the molecular changes occurring in their development; and compared to other malignancies, they are associated with a high rate of somatic mutations [20]. Therefore, characterising molecular changes allows implementation of individualised clinical approach, and it may have prognostic significance.

There are 3 levels within a cell where defined genetic changes may occur. The first level is called the input layer and it is integrated into the cytoplasmic membrane, which consists of ligands and surface receptors. These are for example the receptor tyrosine kinases (RTKs), including KIT and ALK. After the receptors are activated, the next level is launched, i.e., signal transduction pathways. This level consists of two main pathways: MAPK (mitogen-activated protein kinase) and PI3K / AKT / mTOR (phosphatidylinositol-3-kinase pathway). The signalling cascade ends at the last effector level in the cell nucleus (e.g., the TERT gene) with the activation or inhibition of transcription factors [20].

One of the most commonly observed and characteristic pathomechanisms in melanomas involves activation of the MAPK pathway with its main components of RAS / RAF / MEK / ERK kinases. This activation occurs as a result of mutations in the genes which encode proteins involved in the signalling pathway. The most commonly mutated proto-oncogenes are BRAF and NRAS.

\section{BRAF}

BRAF gene (B-Raf proto-oncogene, serine / threonine kinase), located on the long arm of chromosome 7 (7q34), encodes the serine-threonine kinase which activates the ERK pathway. BRAF mutations are seen in approximately $50 \%$ of advanced melanoma cases and are common in patients with no history of sun damage to the skin. These mutations are very rarely found in melanomas of the mucous membranes or oral cavity [21]. Despite identification of many different mutations in the two segments of the kinase domain, the most common one is a substitution of valine for another amino acid at position 600 of the amino acid chain (more than 97\% of the mutations) [22]. The most common change involves substitution of glutamic acid (V600E, 70-80\%). This, in turn, activates BRAF and causes a more than 800 -fold increase of the phosphorylation capacity of the substrate MEK [23].

The next most common alteration concerns V600K (lysine substitution, 10-20\%). Less common changes are V600R, V600D, and V600M (substitutions for arginine, aspartic acid, and methionine, respectively) [21]. BRAF mutation leads to an increase in the cell proliferation index independent of external signals (activation of the MAPK / ERK pathway).

Melanomas with the BRAF mutation have poor prognosis. The disease has an aggressive course associated with shorter survival time in patients with high (IV) stage cancer compared to patients without the BRAF mutation (wild type - WT). Further, $B R A F+$ melanomas are more common in younger people and, unlike wild-type melanomas, they are characterised by superficial tumour spread or nodular type [22]. Mutations of the BRAF gene in melanoma always coexist with inactivation of the suppressor gene, e.g. PTEN or TP53 (oncogene / tumour suppressor gene effect) [24]. 


\section{NRAS}

NRAS gene (NRAS proto-oncogene, GTPase) encoding small-molecule GTPase is located on the short arm of chromosome 1 (1 1 13.2). NRAS is the next most commonly mutating protein in melanomas, and the MAPK pathway is one of its several effector pathways. It is estimated that approximately $15-25 \%$ of melanoma cases have activating mutations in NRAS [25]. The most common NRAS mutation in melanoma is substitution of glutamine with other amino acids at codon 61 (Q61). Typically, these are arginine $(R)$, leucine $(L)$, lysine $(K)$ and histidine $(H)$ [26]. NRAS and BRAFV600 mutations are mutually exclusive. Inactivation of $\mathrm{p} 53$ or 16 and coexistence of the NRAS mutation are factors that trigger the process of neoplastic transformation [24]. The NRAS protein is a GTPase responsible for the hydrolysis of GTP to GDP. Mutations commonly found in melanoma interfere with the hydrolysis process and NRAS is permanently bound to GTP, causing its continued activity independent of external signals. NRAS activates the MAPK pathway by CRAF kinase (BRAF independent pathway activation), which translates into increased proliferation. In addition, it also activates the PI3K / AKT pathway. This, in turn, is associated with modulating the growth and survival of cancer cells [27].

The NRAS mutations are most common in elderly patients who are chronically exposed to UV [28]. Presence of a NRAS mutation is an independent negative prognostic marker associated with higher risk of nodal metastases and lower median survival compared to patients without this change [29].

\section{NF1}

In 2015, The Cancer Genome Atlas (TCGA) published a sequence analysis of the exome in 333 patients with primary and/or metastatic melanoma. The data showed that skin melanomas can be divided into 4 genomic subgroups, which include cancers:

- with BRAF mutation,

- with NRAS mutation,

- with NF1 (neurofibromin 1),

- triple wild type, i.e. tumours without mutations in the above genes [30].

However, clinical implications concerning prognosis and forecasting response to treatment are still equivocal with respect to the group with NF1 gene mutations. Therefore, further studies are necessary to introduce guidelines for management of patients with this mutation [8].

\section{PTEN}

PTEN gene (phosphatase and tensin homolog), located on the long arm of the chromosome 10 (10q23.31), encodes phosphatase which acts as a tumour successor by blocking the PI3K signalling pathway through lipid phosphatase activity and by negatively regulating the MAPK pathway through protein phosphatase activity (double specificity).
In about half of melanomas with BRAF mutations, loss of expression of PTEN protein is detected. This loss reflects homozygous deletion of the gene or other genetic and epigenetic changes which lead to reduction/loss of protein expression. Further, a subgroup of melanomas can be identified with amplification of AKT3 (AKT serine/threonine kinase 3) - effector of PI3K pathway. This amplification is an independent mechanism that leads to activation of the PI3K pathway in tumours with present mutations that activate BRAF [31]. The consequences of the loss of PTEN function associated with AKT3 amplification still need to be clarified. However, it is suggested that activation of the PI3K pathway affects the expression of the porapoptotic protein BCL2L11. Lack of PTEN activity inhibits expression of BCL2L11, which translates into increased resistance of cells to apoptosis. The moment of loss of PTEN activity remains unresolved - whether it occurs in the initial or later stages of carcinogenesis [31].

\section{KIT}

Mutations which lead activation and/or amplification of KIT (KIT proto-oncogene, receptor tyrosine kinase), are relatively common in rare (1-3\% of all melanoma cases) melanomas of the mucous membranes and subungual tissues (10-40\%). Further, unlike the BRAF mutation, they occur in people who are chronically exposed to skin damage caused by solar radiation [32, 33]. Mutations / amplifications of the KIT protein lead to constitutive activation of various intracellular pathways, including MAPK / ERK and PI3K / AKT, which play key roles in melanoma development. KIT gene mutations in melanoma are most commonly (about 70\%) located in:

- exon 11 - most commonly substitution of proline for leucine in codon 576 (L576P), and

- exon 13 - most commonly substitution of glutamic acid for lysine in codon 642 (K642E) [33].

These mutations cause enhanced proliferation which translates to increased expression of Ki-67 protein (proliferation biomarker) in immunohistochemistry testing of patients with a mutation as compared to patients with the unchanged gene. The presence of a mutation in the KIT gene is a prognostic marker associated with a worse prognosis as compared to melanomas without this change [34].

\section{GNAQ/GNA11 and BAP1}

The genetic profile of uveal melanomas turned out to be completely different from that of the skin or mucous membranes melanomas, because in these cancers there are no mutations of proto-oncogenes and suppressor genes crucial for development of skin melanomas. However, they have characteristic mutations in two proto-oncogenes: GANQ (G protein subunit alpha q) and GNA11 (G protein subunit alpha 11) which are mutually exclusive. Both genes encode the a-subunit of a G protein with GTPase activity involved in the activation of various signal transmission pathways [35]. Mutations of these 
genes lead to the inactivation of the GTPase function. This is associated with constitutive binding of protein with GTP and similar as in the case of NRAS - it leads to its constant activity.

Further, beside mutations of the listed protooncogenes, point mutations of the BAP1 suppressor protein-encoding gene were found in uveal melanomas. The biggest number of mutations are present in the domains binding BAP1 to BRCA1 and BARD1 $[19,35,36]$.

\section{Genetic analysis of somatic changes}

Somatic changes are characteristic and present only in the patient's cancer cells. Their identification allows introduction of treatment targeted at these changes. Consequently, the applied therapy may be much more effective than the classic chemotherapy.

The most commonly analysed material is DNA isolated from paraffin embedded tissue blocks. The key step before isolating the genetic material is to assess the percentage of neoplastic cells (which should be higher than 50\%). This is a precondition for selection of the tissue fragment to be tested. BRAF V600 mutation status is the only biomarker currently considered important in the treatment of advanced metastatic cutaneous melanoma. Therefore, assessment of this status has become a priority in selecting therapy and has been included in guidelines by both the European Society for Medical Oncology (ESMO) and American National Comprehensive Cancer Network (NCCN) [17, 37, 38]. So far, several methods have been developed that can be used to detect BRAF mutations. These include:

- Sanger sequencing, immunohistochemistry (IHC),

- pyrosequencing,

- mutation-specific PCR,

- mutation specific real-time PCR / qPCR,

- digital PCR,

- high-resolution melting curve analysis (HRM),

- next-generation sequencing (NGS) [39].

Recommendations for identification of BRAF mutations in clinical practice indicate sequential analysis using two methods. The first step is to perform screening by IHC with monoclonal VE1 antibodies (specific for the mutant version of the BRAF protein with the V600E mutation). Secondly, the presence of the mutation must be confirmed by one of the methods of molecular biology. These recommendations are associated with the risk of false negative results and/or failure to detect presence of other mutations than V600E by IHC. If there is not enough material for genetic testing, then $\mathrm{IHC}$ remains the method of choice.

It should be remembered that sensitive molecular methods, e.g., real-time PCR may detect BRAF mutations which occur in a small percentage of tumour cells (even $>5 \%$ ), mostly wild type. However, it is not actually of clinical relevance in response to targeted therapy. Therefore, it seems that the NGS method is currently the best molecular method [39]. It allows simultaneous analysis of all genes which are relevant in mela- noma (there are commercially available panels), and apart from high sensitivity, it indicates the percentage of mutated alleles.

Nowadays in Poland and worldwide, there are many commercial tests available which allow fast and unequivocal determination of BRAF mutation status. Most commonly, these are tests based on real-time PCR and - more importantly - optimised for DNA from paraffin embedded tissue blocks. Reference laboratories should use tests certified for diagnostic purposes (e.g., CE IVD certificate or recommendations by the American Food and Drug Administration [FDA]). Further, to ensure high level of performance of the tests, such laboratories should regularly undergo international inspections for external quality control. They should also promptly implement the latest recommendations.

Liquid biopsy is another way to obtain material for tests. It is gaining clinical significance with respect to analysing mutations in neoplastic tumours (especially inoperable ones), as well as treatment and resistance monitoring. This method involves identification of circulating tumour cells (CTCS), circulating tumour DNA ( ctDNA) or circulating tumour RNA (ctRNA) in the patient's blood, as they hold characteristic mutations of prognostic importance [40].

\section{Conflict of interest: none declared}

\author{
Justyna Gil \\ Wroclaw Medical University \\ Faculty of Medicine \\ Chair and Department of Genetics \\ ul. Marcinkowskiego 1 \\ 50-368 Wrocław \\ e-mail:justyna.gil@umed.wroc.pl
}

Received: 24 Mar 2021

Accepted: 25 Mar 2021

\section{References}

1. Sąsiadek M, Łaczmańska I, Maciejczyk A, et al. Fundamentals of personalised medicine in genetic testing-based oncology. Nowotwory. Journal of Oncology. 2020; 70(4): 144-149, doi: 10.5603/njo.2020.0029.

2. Shain $A H$, Bastian BC. From melanocytes to melanomas. Nat Rev Cancer. 2016; 16(6): 345-358, doi: 10.1038/nrc.2016.37, indexed in Pubmed: 27125352.

3. http://onkologia.org.pl/wp-content/uploads/Nowotwory_2017.pdf (28.01.2021).

4. Michalska-Jakubus M. Malignant melanoma - epidemiology, etiopathogenesis and prognosis. Med Rodz. 2006(May 15).

5. Elwood J, Jopson J. Melanoma and sun exposure: An overview of published studies. International Journal of Cancer. 1997; 73(2): 198-203, doi: 10.1002/(sici)1097-0215(19971009)73:2<198::aid-ijc6>3.0.co;2-r.

6. Rastrelli M, Tropea S, Rossi CR, et al. Melanoma: Epidemiology, risk factors, pathogenesis, diagnosis and classification. In Vivo. International Institute of Anticancer Research. 2014; 28. https://moh-it.pure. elsevier.com/en/publications/melanoma-epidemiology-risk-factorspathogenesis-diagnosis-and-cla (1.03.2021).

7. Rutkowski P, Wysocki PJ, Nasierowska-Guttmejer A, et al. Cutaneous melanomas. Oncol Clin Pract. 2020; 16(4): 163-182, doi: 10.5603/ OCP.2020.0021.

8. Garbe C, Amaral T, Peris K, et al. European consensus-based interdisciplinary guideline for melanoma. Part 1: Diagnostics - Update 2019. European Journal of Cancer. 2020; 126: 141-158, doi: 10.1016/j. ejca.2019.11.014 
9. Soura E, Eliades P, Shannon K, et al. Hereditary melanoma: Update on syndromes and management. J Am Acad Dermatol. 2016; 74(3): 395-407, doi: 10.1016/j.jaad.2015.08.038.

10. Gapska P, Scott RJ, Serrano-Fernandez P, et al. CDKN2A common variants and their association with melanoma risk: a population-based study. Cancer Res. 2005; 65(3): 835-839, indexed in Pubmed: 15705881.

11. DebniakT, Górski B, Huzarski T, et al. A common variant of CDKN2A (p16) predisposes to breast cancer. J Med Genet. 2005; 42(10): 763-765, doi: 10.1136/jmg.2005.031476, indexed in Pubmed: 15879498.

12. Debniak T, Scott RJ, Huzarski T, et al. CDKN2A common variant and multi-organ cancer risk--a population-based study. Int J Cancer. 2006; 118(12): 3180-3182, doi: 10.1002/ijc.21760, indexed in Pubmed: 16395703.

13. Takebe H, Nishigori C, Tatsumi K. Melanoma and Other Skin Cancers in Xeroderma Pigmentosum Patients and Mutation in Their Cells. Journal of Investigative Dermatology. 1989; 92(5): S236-S238, doi: 10.1038/jid.1989.73.

14. Góralska A, Błaszczyk J. Znamię atypowe, znamię dysplastyczne, zespółznamion atypowych-kontrowersje nomenklaturowe, trudności diagnostyczne i znaczenie prognostyczne Atypical naevus, dysplastic naevus, dysplastic naevus syndrome-nomenclature controversy, diagnostic difficulties and prognostic perspectives. Dermatology Review. 2013; 100.

15. Lynch HT, Shaw TG. Familial atypical multiple mole melanoma (FAMMM) syndrome: history, genetics, and heterogeneity. Fam Cancer. 2016; 15(3): 487-491, doi: 10.1007/s10689-016-9888-2, indexed in Pubmed: 26892865.

16. Nieuwenburg SA, Adan F, Ruijs MWG, et al. Cumulative risk of skin cancer in patients with Li-Fraumeni syndrome. Fam Cancer. 2020; 19(4): 347351, doi: 10.1007/s10689-020-00178-1, indexed in Pubmed: 32356166.

17. Swetter SM, Tsao H, Bichakjian CK, et al. Guidelines of care for the management of primary cutaneous melanoma. J Am Acad Dermatol. 2019; 80(1): 208-250, doi: 10.1016/j.jaad.2018.08.055, indexed in Pubmed: 30392755

18. Leachman SA, Lucero OM, Sampson JE, et al. Identification, genetic testing, and management of hereditary melanoma. Cancer Metastasis Rev. 2017; 36(1): 77-90, doi: 10.1007/s10555-017-9661-5, indexed in Pubmed: 28283772.

19. Casula M, Paliogiannis P, Ayala F, et al. Melanoma Unit of Sassari (MUS), Italian Melanoma Intergroup (IMI). Germline and somatic mutations in patients with multiple primary melanomas: a next generation sequencing study. BMC Cancer. 2019; 19(1): 772, doi: 10.1186/s12885019-5984-7, indexed in Pubmed: 31382929.

20. Helgadottir H, Rocha Trocoli Drakensjö I, Girnita A. Personalized Medicine in Malignant Melanoma: Towards Patient Tailored Treatment. Front Oncol. 2018; 8: 202, doi: 10.3389/fonc.2018.00202, indexed in Pubmed: 29946532.

21. Ascierto PA, Kirkwood JM, Grob JJ, et al.The role of BRAF V600 mutation in melanoma. JTransI Med. 2012; 10: 85, doi: 10.1186/1479-5876-10-85, indexed in Pubmed: 22554099.

22. Cheng L, Lopez-Beltran A, Massari F, et al. Molecular testing for BRAF mutations to inform melanoma treatment decisions: a move toward precision medicine. Mod Pathol. 2018; 31(1): 24-38, doi: 10.1038/ modpathol.2017.104, indexed in Pubmed: 29148538.

23. Mikula $\mathrm{H}$, Stapleton $\mathrm{S}$, Kohler $\mathrm{RH}$, et al. Design and Development of Fluorescent Vemurafenib Analogs for Imaging. Theranostics. 2017; 7(5): 1257-1265, doi: 10.7150/thno.18238, indexed in Pubmed: 28435463.

24. Palmieri G, Colombino M, Casula $\mathrm{M}$, et al. Italian Melanoma Intergroup (IMI). Molecular Pathways in Melanomagenesis: What We Learned from
Next-Generation Sequencing Approaches. Curr Oncol Rep. 2018; 20(11): 86, doi: 10.1007/s11912-018-0733-7, indexed in Pubmed: 30218391.

25. Gutiérrez-Castañeda LD, Nova JA, Tovar-Parra JD. Frequency of mutations in BRAF, NRAS, and KIT in different populations and histological subtypes of melanoma: a systemic review. Melanoma Res. 2020; 30(1): 62-70, doi: 10.1097/CMR.0000000000000628, indexed in Pubmed: 31274706 .

26. Hélias-Rodzewicz Z, Funck-Brentano E, Terrones N, et al. Variation of mutant allele frequency in NRAS Q61 mutated melanomas. BMC Dermatol. 2017; 17(1): 9, doi: 10.1186/s12895-017-0061-x, indexed in Pubmed: 28668077.

27. Fedorenko IV, Gibney GT, Smalley KSM. NRAS mutant melanoma: biological behavior and future strategies for therapeutic management. Oncogene. 2013; 32(25): 3009-3018, doi: 10.1038/onc.2012.453, indexed in Pubmed: 23069660.

28. Muñoz-Couselo E, Adelantado EZ, Vélez CO, et al. NRAS-mutant melanoma: current challenges and future prospect. OncoTargets and Therapy. 2017; Volume 10: 3941-3947, doi: 10.2147/ott.s117121.

29. Jakob JA, Bassett RL, Ng CS, et al. NRAS mutation status is an independent prognostic factor in metastatic melanoma. Cancer. 2012; 118(16): 4014-4023, doi: 10.1002/cncr.26724, indexed in Pubmed: 22180178.

30. Akbani R, Akdemir KC, Aksoy BA, et al. Genomic Classification of Cutaneous Melanoma. Cell. 2015; 161(7): 1681-1696.

31. Aguissa-Touré AH, Li G. Genetic alterations of PTEN in human melanoma. Cell Mol Life Sci. 2012; 69(9): 1475-1491, doi: 10.1007/s00018-0110878-0, indexed in Pubmed: 22076652.

32. Ma X, Wu Y, Zhang $T$, et al. The clinical significance of mutations in metastatic oral mucosal melanoma in China. Oncotarget. 2017; 8(47): 82661-82673, doi: 10.18632/oncotarget.19746, indexed in Pubmed: 29137292.

33. Pham DD, Guhan S, Tsao H. KIT and Melanoma: Biological Insights and Clinical Implications. Yonsei Med J. 2020; 61(7): 562-571, doi: 10.3349/ ymj.2020.61.7.562, indexed in Pubmed: 32608199.

34. Pracht $M$, Mogha A, Lespagnol A, et al. Prognostic and predictive values of oncogenic BRAF, NRAS, C-KIT and MITF in cutaneous and mucous melanoma. J Eur Acad Dermatol Venereol. 2015; 29(8): 1530-1538, doi: 10.1111/jdv.12910, indexed in Pubmed: 25623140.

35. Sheng $X$, Kong $Y$, Li Y, et al. GNAQ and GNA11 mutations occur in 9.5\% of mucosal melanoma and are associated with poor prognosis. Eur J Cancer. 2016; 65: 156-163, doi: 10.1016/j.ejca.2016.06.019, indexed in Pubmed: 27498141.

36. Livingstone E, Zaremba A, Horn S, et al. GNAQ and GNA11 mutant nonuveal melanoma: a subtype distinct from both cutaneous and uveal melanoma. Br J Dermatol. 2020; 183(5): 928-939, doi: 10.1111/ bjd.18947, indexed in Pubmed: 32064597.

37. Michielin O, van Akkooi A, Lorigan P, et al. ESMO consensus conference recommendations on the management of locoregional melanoma: under the auspices of the ESMO Guidelines Committee. Ann Oncol. 2020; 31(11): 1449-1461, doi: 10.1016/j.annonc.2020.07.005, indexed in Pubmed: 32763452.

38. Coit DG, Thompson JA, Albertini MR, et al. Cutaneous melanoma, version 2. JNCCN. 2019; 17: 367-402.

39. Vanni I, Tanda ET, Spagnolo F, et al. The Current State of MolecularTesting in the BRAF-Mutated Melanoma Landscape. Front Mol Biosci. 2020; 7: 113, doi: 10.3389/fmolb.2020.00113, indexed in Pubmed: 32695793.

40. Marczynski GT, Laus AC, Dos Reis MB, et al. Circulating tumor DNA (ctDNA) detection is associated with shorter progression-free survival in advanced melanoma patients. Sci Rep. 2020; 10(1): 18682, doi: 10.1038/ s41598-020-75792-1, indexed in Pubmed: 33122747. 